\title{
Treadmill training as an augmentation treatment for Alzheimer's disease: a pilot randomized controlled study
}

Treinamento na esteira como um tratamento adicional para a doença de Alzheimer: estudo piloto controlado randomizado

Cynthia Arcoverde ${ }^{1}$, Andrea Deslandes ${ }^{2}$. Helena Moraes ${ }^{3}$, Cloyra Almeida ${ }^{4}$, Narahyana Bom de Araujo ${ }^{5}$, Paulo Eduardo Vasques ${ }^{6}$, Heitor Silveira', Jerson Laks $^{8}$

\begin{abstract}
Objective: To assess the effect of aerobic exercise on the cognition and functional capacity in Alzheimer's disease (AD) patients. Method: Elderly $(n=20)$ with mild dementia (NINCDS-ADRDA/CDR1) were randomly assigned to an exercise group (EG) on a treadmill (30 minutes, twice a week and moderate intensity of $60 \% \mathrm{VO}_{2 \text { max }}$ ) and control group (GC) 10 patients. The primary outcome measure was the cognitive function using Cambridge Cognitive Examination (CAMCOG). Specifics instruments were also applied to evaluate executive function, memory, attention and concentration, cognitive flexibility, inhibitory control and functional capacity. Results: After 16 weeks, the EG showed improvement in cognition CAMCOG whereas the CG declined. Compared to the CG, the EG presented significant improvement on the functional capacity. The analysis of the effect size has shown a favorable response to the physical exercise in all dependent variables. Conclusion: Walking on treadmill may be recommended as an augmentation treatment for patients with AD.
\end{abstract}

Keywords: Alzheimer's disease, aerobic exercise, physical training, cognitive function, functional capacity.

RESUMO

Objetivo: Avaliar o efeito do exercício aeróbio na cognição e na capacidade funcional em pacientes com Doença de Alzheimer (DA). Método: Idosos ( $n=20)$ com demência leve ((NINCDS-ADRDA/CDR1) foram randomizados em grupo exercício (GE) na esteira (30 minutos, 2 vezes por semana e intensidade moderada de $60 \% \mathrm{VO}_{2 \text { max }}$ ) grupo controle (GC) 10 pacientes. A medida principal foi a função cognitiva através do Cambridge Cognitive Examination (CAMCOG). Instrumentos específicos também foram aplicados para avaliar a função executiva, atenção e concentração, flexibilidade cognitiva, controle inibitório e capacidade funcional. Resultados: Após 16 semanas, o GE mostrou melhora na cognição CAMCOG enquanto o CG declinou. Comparado ao GC, o GE apresentou melhora significativa na capacidade funcional. A análise do tamanho de efeito mostrou resposta favorável do exercício físico em todas as variáveis dependentes. Conclusão: Caminhar na esteira pode ser recomendado como um tratamento adicional para pacientes com doença de Alzheimer.

Palavras-chave: doença de Alzheimer, exercício aeróbio, treinamento físico, função cognitiva, capacidade funcional.

The state of the art with regard to the neurodegenerative dementias, especially for Alzheimerás disease $(\mathrm{AD})$, is that their progression is still irreversible causing cognitive, motor, and behavioral impairment. Although the current pharmacological treatments attenuate cognitive decline in some cases, the most treatments do not avoid the

\footnotetext{
${ }^{1}$ Physical therapist. MSc in Mental Health, Instituto de Psiquiatria, Universidade Federal do Rio de Janeiro, Rio de Janeiro RJ, Brazil; ${ }^{2}$ Coordinator, Laboratório de Neurociência do Exercício; Researcher, Fundação de Amparo à Pesquisa do Estado do Rio de Janeiro, Rio de Janeiro RJ, Brazil; ${ }^{3} \mathrm{MSc}$ in Mental Health, Instituto de Psiquiatria, Universidade Federal do Rio de Janeiro, Rio de Janeiro RJ, Brazil;

${ }^{4} \mathrm{MD}$, Instituto de Psiquiatria, Universidade Federal do Rio de Janeiro, Rio de Janeiro RJ, Brazil;

${ }^{5}$ MSc Psychologist, Instituto de Psiquiatria, Universidade Federal do Rio de Janeiro, Rio de Janeiro RJ, Brazil;

${ }^{6}$ Physical therapist, MSc in Mental Health, Instituto de Psiquiatria, Universidade Federal do Rio de Janeiro, Rio de Janeiro RJ, Brazil;

${ }^{7} \mathrm{MSc}$ in Mental Health, Instituto de Psiquiatria, Universidade Federal do Rio de Janeiro, Rio de Janeiro RJ, Brazil;

${ }^{8}$ Instituto de Psiquiatria, Universidade Federal do Rio de Janeiro; Centro de Estudo e Pesquisa do Envelhecimento - Instituto Vital Brazil; Researcher Pq2 of the Brazilian National Research Council.

Correspondence: Jerson Laks; Av. Nossa Senhora de Copacabana, 749/802; 22050-000, Rio de Janeiro RJ, Brazil; E-mail: jlaks@centroin.com.br

Support: This study was supported by Brazilian National Research Council (Conselho Nacional de Pesquisa-CNPq) [grant \# 300411/2009-0] and Fundação de Amparo à Pesquisa do Estado do Rio de Janeiro. [\#E-26/102.022/2009; \# E-26/102.174/2013].

Conflict of interest: There is no conflict of interest to declare.

Received 30 June 2013; Received in final form 23 October 2013; Accepted 30 October 2013.
} 
motor and functional changes caused by the progress of the disease ${ }^{1}$.

However, there is sufficient evidence that an active lifestyle may be associated to the prevention of dementia as well as it may be an important aid for the treatment of the syndrome ${ }^{2,3}$. Compared to sedentary people, the individuals who practice physical exercises present a lower chance of developing dementia (28\%) and $\mathrm{AD}(45 \%)^{3}$. Our laboratory has observed a positive correlation between improvement in activities of daily living (ADL) and in the overall cognitive state among people with dementia who still have an active lifestyle ${ }^{4}$. Moreover, there is increasing evidence to support an inverse relationship between cardiorespiratory fitness and brain atrophy in the earliest clinical stages of $\mathrm{AD}$. Higher fitness levels in early $\mathrm{AD}$ patients were associated with preserved brain volume (less brain atrophy) independent of age ${ }^{5}$.

Several studies have shown that physical training programs may have a role in the improvement of ADL, quality of life, depressive symptoms, and motor and cognitive function in patients with $\mathrm{AD}^{3,4,6-11}$. However, some methodological questions may affect the application of the training protocols. The use of recognized intensity patterns like maximum oxygen uptake $\left(\mathrm{VO}_{2 \max }\right)$ andmaximum heart rate $\left(\mathrm{HR}_{\max }\right)$ percentage to control the training of patients with dementia is still rare in the literature. Therefore, these issues seem to hamper a proper definition of an ideal exercise prescription. Whereas studies which used walking as a type of aerobic intervention in patients with dementia did not provide the same results ${ }^{8,12,13}$. In recent years, several systematic reviews have observed positive effects of physical exercise on people with dementia ${ }^{3,9}$. However, according to Forbes et al. ${ }^{14}$ there is insufficient evidence to determine the effectiveness of physical activity programs in managing or improving cognition. In a recent systematic review, Littbrand et al. ${ }^{9}$ concluded that low methodological quality and/or an intervention of insufficient intensity can be responsible for the exercise has no effect on cognitive functions among people with dementias.

These effects are dependent, however, on the intensity and frequency of exercise. There is an inverted- $U$ relation between the effect of physical exercise and cognition and mental health, with the best results associated to the moderate intensity effort ${ }^{15}$. According to Radak et al. ${ }^{15}$ although exercise is a stressor itself, it may also contribute to protect the body from stronger stressors when in moderate quantities. Following this line of reasoning, the present study aimed at assessing the effect of moderate aerobic exercise on cognition and functional abilities in $\mathrm{AD}$ patients. Although previous studies have investigated the effect of exercise in $\mathrm{AD}$ patients, the present study is the first article investigating the effect of exercise using treadmill with controlled intensity

\section{METHOD}

\section{Participants}

We carried out a four-month controlled, randomized, and simple-blind pilot study. The patients were recruited by trained psychiatrists from the Center for Alzheimer's disease in the Institute of Psychiatry of the Federal University of Rio de Janeiro (IPUB/UFRJ). They were randomized with a blind design to an exercise group (EG) and control group (CG) by a researcher (D.A) who did not participate of the initial assessments.

A total of 20 elderly with dementia were included in the study, being 16 patients diagnosed with $\mathrm{AD}$ and four with mixed dementia (MD). Inclusion criteria were: 1) diagnosis of $\mathrm{AD}$ and $\mathrm{MD}$ according to the National Institute of Neurological and Communicative Disorders and StrokeAlzheimer's Disease and Related Disorders Association (NINCDS-ADRDA $)^{16}$ and National Institute of Neurological Disorders and Stroke- Association Internationale pour la Recherche et l' Enseignement en Neurosciences (NINDSAIREN) $)^{17}$, respectively; 2) Mini-Mental State Examination (MMSE) score $\geq 15^{18}$; 3) Clinical Dementia Rating (CDR) score 1 (moderate memory loss) ${ }^{19}$; 4) regular use of anticholinesterase drugs or another type of pharmacological treatment to $\mathrm{AD}$ for at least six months; 5) cardiologist's authorization; 6) at least six months without practicing physical exercises. The patients were excluded from the research if they presented: 1) clinical depression or Cornell Scale $\geq 7^{20}$; 2 ) other types of dementia; 3) physical limitation due to other pathologies or associated neurological disease; 4) severe or uncontrolled arterial hypertension; 5) marked visual and/or auditory deficit; 6) incapacity to perform physical exercise due to neurological or neuromuscular impairments; 7) illiteracy; 8) less than six months of treatment at the outpatient unit.

\section{Intervention}

Physical exercise program

The EG was composed by ten patients $(\mathrm{AD}=8 ; \mathrm{MD}=2)$ who were submitted to a period of adaptation to the treadmill (BH fitness- Explorer Pro Action) for four weeks, with progressive intensity and duration, until reaching a percentage of $40 \% \mathrm{VO}_{2 \max }$ (initial speed $2 \mathrm{~km} / \mathrm{h}$, initial duration 20 minutes). During this period, the patients got familiarized with the Borg's perceived exertion scale. After the adaptation period, the patients were submitted to treadmill walking for 30 minutes twice a week for three months. The intervention was divided into three phases: 1) Warm-up exercises on the treadmill for ten minutes at an intensity of $40 \% \mathrm{VO}_{2 \max }$; 2) 20 minutes at an intensity of $60 \% \mathrm{VO}_{2 \max }$; 3) 5 minutes of supervised stretching exercises focused on the big muscles groups. The patients were supervised by physical therapist and physical educators, and monitored every five 
minutes by frequency counters (Polar ${ }^{\mathbb{B}}$ display) and Borg Scale. Blood pressure was checked before and immediately after each exercise session. Moreover, some instructions were used to them, such as to look in front, to hold on the treadmill, to give wide steps, do not talk, to keep adequate posture.

\section{Control Group}

The CG was composed by ten patients $(A D=8 ; M D=2)$ who maintained only the clinical and pharmacological treatment along the four months of follow-up. These patients attending the hospital only to medical routine and did not do another type of intervention during this period.

\section{MEASURES}

\section{Cognitive assessment}

For the neuropsychological assessment, the general cognitive state was performed with the Brazilian validated version of the Cambridge Cognitive Examination (CAMCOG) $)^{21}$ (CANDEX - Cambridge Examination for Mental Disorders of the Elderly) and is subdivided in scales which assess orientation, expressive and comprehensive language, memory (including remote memory, recent memory and learning processes), attention, praxis, calculation, abstract thinking and perception. The Clock Drawing Test (CDT) was used to evaluate executive function, as part of CAMCOG. The verbal fluency test (animal category) was used as a measure of the executive, semantic memory and language functions ${ }^{23}$. For the learning and verbal memory evaluation, the sum of the Al-A5 list of the Rey Auditory Verbal Learning Test (RAVLT) was used ${ }^{23}$. The Digit Span, a subtest of the WAIS-R Scale ${ }^{22}$, was used to evaluate immediate memory and attention. The Trail Making Test A was also used to evaluate the executive function, whereas selective attention, mental flexibility and inhibitory control were evaluated by the Stroop test ${ }^{23}$.

\section{FUNCTIONAL CAPACITY}

Static and dynamic balance: Berg Balance Scale (BERG) $)^{24}$ is composed by 14 tasks related to daily life and each item has one ordinal scale of five alternatives which vary from 0 to 4 points, with a total of 56 points. The higher the score, the better is the performance. Task 13 corresponds to Functional Reach Test (FR), which is clinically useful to identify the fall risk.

Mobility: The "Timed Up and Go" test (TUGT) ${ }^{25}$ and its modified version "Modified Timed up and go" (TUG mod) have been used with the objective of evaluating the functional mobility and the dual-task ability, respectively. In the first test, the patient walks three meters while the time is counted, and, in the second one, the patient is asked to walk and evoke names of animals. More than 10 seconds is considered risk of functional loss.

Lower limb strength: The Sit-to-Stand test (STS) ${ }^{25}$ counts the number of times the patient can sit down and stand up in 30 seconds. This test evaluates lower limb strength and it is associated to the cardiovascular capacity of elderly people.

Ergometric test and rest electrocardiogram (ECG): All patients were submitted to a rest ECG for the detection of a possible alteration on the cardiac rhythm, using the three-channel electrocardiograph EP-3 Dixtal. For obtaining maximum values of oxygen uptake $\left(\mathrm{VO}_{2 \max }\right)$, the adapted Bruce treadmill protocol was used. The data were analyzed by the TEB digital program (Brazilian Electronic Technology). The required procedures were 3 minutes of warm-up with initial speed of $2.5 \mathrm{~km} / \mathrm{h}, 0 \%$ of inclination and 3 minute-stages with gradual and slight increases of speed and/or inclination.

\section{STATISTICAL ANALYSIS}

The Shapiro-Wilk and Levine tests were used with the purpose of verifying the normality and homoscedasticity, respectively. At baseline, the differences between groups were assessed by an independent sample $t$-test (educational level, MMSE, CAMCOG, RAVLT, duration of disease, FR, TUGT and STS) or a Mann-Whitney U-test (age, body mass index, number of comorbidities and falls, CDT, Trail Making Test A, depressive symptoms, Stroop test, forward and backward Digit Span, TUGTmod and BERG). The after intervention delta (after intervention minus baseline) was calculated so as to compare the two groups and the two moments. The statistical significance of this comparison was assessed by using an independent sample $t$-test for the variables classified as parametric (MMSE, CAMCOG, CDT, STROOP, Digit Span DO and IO, FR, TUGTmod and STS) and by a Mann-Whitney U-test for the non-parametric variables (Verbal Fluency Test, Trail Making Test A, BERG and TUGT).

An effect size (ES) analysis was performed to determine the differences between the two groups. ES was calculated as the differences of the two means (before intervention (M2) - after intervention (M1) divided by the pooled standard deviation $\left(\mathrm{SD}_{\text {pooled }}\right)(\mathrm{ES}=\mathrm{M} 2-\mathrm{M} 1 / \mathrm{SD})$. For a better interpretation of the results, the result signs were modified to characterize the clinical improvement as a positive result. The SPSS ${ }^{10}$ software (version 17.0) was used for data analysis and the accepted level of significance was $\mathrm{p} \leq 0.05$.

This study was approved by the Ethics Committee of the Institute of Psychiatry of the Federal University of Rio de 
Table 1. Sample's descriptive analysis.

\begin{tabular}{|c|c|c|c|}
\hline & $\begin{array}{l}\text { Control Group } \\
\qquad(n=10) \\
\text { Mean (SD) }\end{array}$ & $\begin{array}{l}\text { Exercise Group } \\
\qquad(n=10) \\
\text { Mean (SD) }\end{array}$ & $\mathrm{p}$-value \\
\hline Age $\left(\right.$ years) ${ }^{\star}$ & $79(74.7-82.2)$ & $78.5(64-81.2)$ & .44 \\
\hline Gender (\% female) & 50 & 60 & .45 \\
\hline Education (years) & $8.4(4.0)$ & $8.7(4.7)$ & .88 \\
\hline MMSE (score) & $19.9(3.4)$ & $20.4(2.7)$ & .72 \\
\hline CAMCOG-CANDEX (score) & $68.4(12.2)$ & $69.9(10.8)$ & .77 \\
\hline CDR (score) & 1 & 1 & \\
\hline Duration of disease (years) & $4.1(2.1)$ & $4.3(2.8)$ & .34 \\
\hline Cornell Scale (score) & $4(3.1)$ & $2.5(2)$ & .48 \\
\hline$V_{2 \text { máx }}(\mathrm{ml} / \mathrm{kg} / \mathrm{min})^{*}$ & $23.3(14-34.9)$ & $23.3(14-26.2)$ & .57 \\
\hline Number of comorbidities ${ }^{*}$ & $1(0-2)$ & $1(0.75-2.0)$ & .68 \\
\hline Number of falls*+ & $0.5(0-1)$ & $0(0-1)$ & .83 \\
\hline
\end{tabular}

MMSE: Mini-Mental State Examination; CAMCOG: Cambridge Cognitive Examination; CDR: Clinical Demenia Rating; $\mathrm{VO}_{2}$ : Maximum Oxygen Uptake.

Janeiro (ethics number: 60liv2-09B), and all participants signed informed consent forms before any procedures.

\section{RESULTS}

\section{Descriptive characteristics of the sample}

There was no significant difference between the two groups (control group $\mathrm{X}$ exercise group) at baseline with respect to gender $(\mathrm{p}=0.45)$, age $(\mathrm{p}=0.44 ; 95 \% \mathrm{CI}, 64$ to 85$)$, educational level ( $\mathrm{p}=0.88$; $95 \% \mathrm{CI}$, 3 to 16$)$, duration of the disease ( $p=0.34$; $95 \%$ CI, 2 to 7 ), MMSE ( $p=0.72,95 \% C I, 15$ to 25 ), CAMCOG ( $\mathrm{p}=0.77,95 \% \mathrm{CI}, 43$ to 87 ), comorbidities $(\mathrm{p}=0.68$, $95 \% \mathrm{CI}, 0$ to 4 ), number of falls in the last 12 months $(\mathrm{p}=0.83,95 \% \mathrm{CI}, 0$ to 1$)$, cardiorespiratory function $\left(\mathrm{VO}_{2 \text { máx }}\right)$ $(\mathrm{p}=0.57,95 \% \mathrm{CI}, 14$ to 34$)$, and depressive symptoms (CORNELL) ( $\mathrm{p}=0.48,95 \% \mathrm{CI}, 0$ to 6 ). Table presents the descriptive analysis of the sample. Moreover, the groups did not present any statistically significant difference in other cognitive or functional variables (Table 2 and Table 3).

\section{Adherence to the study, safety and dropouts}

Thirty two sessions of physical exercises were conducted, in which eight subjects presented a total adhesion to the study $(100 \%)$ and two subjects participated of 30 sessions (adherence=93.7\%). There were no dropouts along the study. There were no side effects caused by the physical exercise over a period of 16 weeks.

\section{Neuropsychological assessment}

There was a significant statistical difference between the two groups on the general cognitive state CAMCOG $(\mathrm{p}=0.00)$. The delta values (post-intervention subtracted by the pre-intervention) are presented on Table 2. After 16 weeks, the exercise group showed improvement in cognition CAMCOG whereas the sedentary group declined. However, although the CG showed a decline in MMSE and executive function CDT when compared to the EG, this result did not achieve a statistical significance $(\mathrm{p}=0.57)$ and $(\mathrm{p}=0.06)$. The scores on the verbal fluency test, STROOP, and Trail Making Test A were maintained in the EG as compared

Table 2. Baseline and Delta values (post-intevention subctracted by the pre-intervention) between the two groups in the cognitive aspects.

\begin{tabular}{|c|c|c|c|c|c|}
\hline & \multicolumn{2}{|c|}{ Control Group $(n=10)$} & \multicolumn{2}{|c|}{ Exercise group $(n=10)$} & \multirow[b]{2}{*}{$\mathrm{p}$-values } \\
\hline & $\begin{array}{l}\text { Baseline } \\
\text { Mean (SD) }\end{array}$ & $\begin{array}{c}\text { Delta } \\
\text { Mean (SD); Cl (95\%) }\end{array}$ & $\begin{array}{l}\text { Baseline } \\
\text { Mean (SD) }\end{array}$ & $\begin{array}{c}\text { Delta } \\
\text { Mean (SD); Cl (95\%) }\end{array}$ & \\
\hline MMSE (score) & $19.9(3.4)$ & $-2.10(0.8) ;(-6$ to 1$)$ & $20.4(2.7)$ & 0.30 (2.4); (-2 to 4$)$ & 0.05 \\
\hline CAMCOG (score)* & $68.4(12.2)$ & $-6.10(4.3) ;(-14$ to 0$)$ & $69.9(10.8)$ & 6.10 (6.7); (-5 to 22$)$ & 0.00 \\
\hline CDT (score) & $2.1(1.5)$ & $-1.10(1.1) ;(-3$ to 0$)$ & $2.1(1.1)$ & $-0.10(1.1) ;(-2$ to 2$)$ & 0.06 \\
\hline Verbal Fluency $(\text { score })^{\dagger}$ & $11(8-12)$ & $-1.0(-2.2 ;-0.7) ;(-3$ to 2$)$ & $10(7-11)$ & $0.5(-2.2 ; 2.5) ;(-4$ to 5$)$ & 0.20 \\
\hline Trail Making $A(\mathrm{sec})^{\dagger}$ & $117(69-234)$ & $6.5(-11 ; 35) ;(-3$ to 22$)$ & $130(81-28)$ & $-6.0(-25 ; 19) ;(-5$ to 22$)$ & 0.36 \\
\hline Stroop Test (sec) & $1.7(0.8)$ & -0.35 (1.3); (-3 to 1) & $2,2(1,4)$ & 0.81 (1.6); (-1 to 3.5$)$ & 0.10 \\
\hline Digit Span (score) & $8.9(3.4)$ & $-1.90(3.1) ;(-5$ to 4$)$ & $8.4(2.6)$ & $-0.80(1.9) ;(-5$ to 6$)$ & 0.36 \\
\hline Forward digit-span (score) & $4.8(1.8)$ & $-0.10(1.40) ;(-2$ to 2$)$ & $4.3(1.6)$ & $-0.50(1.2) ;(-2$ to 2$)$ & 0.52 \\
\hline Backward digit-span (score) & $4.1(1.9)$ & $-0.90(1.5) ;(-2$ to 1$)$ & $4(1.9)$ & $-0.70(0.9) ;(-4$ to 1$)$ & 0.73 \\
\hline RAVLT (score) & $20.2(7.1)$ & $-1.30(3.5) ;(6$ to 4$)$ & $20(4.3)$ & $-0.20(4.2) ;(5$ to 9$)$ & 0.53 \\
\hline
\end{tabular}

Note: SD: Standard Deviation. IC: Interval confiance. * Significant difference between the two groups after intervention ( $p=0.05)$. ${ }^{\dagger}$ Median $(25$ and $75 \%$ quartile values). MMSE: Mini-Mental State Examination; CAMCOG: CAMCOG-CAMDEX: Cambridge Examination for Mental Disorders of the Elderly; CDT: Clock Drawing Test; RAVLT: Rey's Auditory Verbal Learning Test. 
Table 3. Baseline and Delta values (post-intervention subtracted by the pre-intervention) between the two groups in the functional capacity.

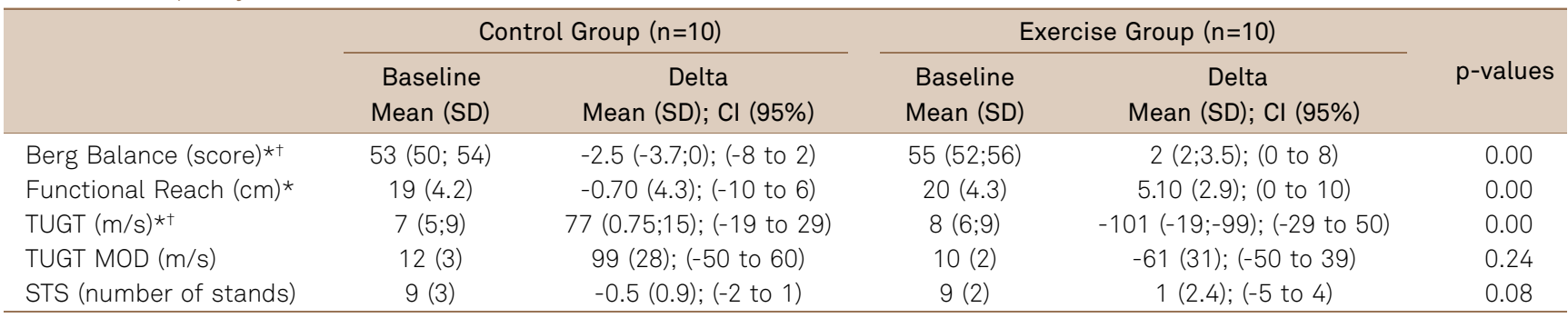

†Median (25; 75\% quartile values). * Significant difference between the two groups after intervention ( $\mathrm{p} \leq 0.05)$. SD: Standard Deviation. Significance $p \leq 0.05$. BERG: Berg Balance Scale; TUGT: Timed Up \& Go Test; TUGTMod: Timed Up \& Go Test Modified. STS: Sit-to-Stand.

to the CG, respectively. Moreover, both groups presented a decline on the forward and backward digit-span test and on the RAVLT.

\section{Functional Capacity Evaluation}

There was a statistically significant difference in the BERG $(\mathrm{p}=0.00)$ and in the FR $(\mathrm{p}=0.00)$ between the two groups. The EG showed increased scores (improvement) whereas the CG declined in all tests. With regard to mobility, there was a significant difference between the groups in the TUGT $(\mathrm{p}=0.00)$. The exercise group walked faster than the sedentary group. However, when we added the task of evoking names of animals (TUGTmod) there was no significant difference between the two groups even though the exercise group presented a better result ( $\mathrm{p}=0.24)$. On the other hand, we did not observe any statistically significant difference between the groups in the STS $(\mathrm{p}=0.08)$. Table 3 shows the comparison between the moments (delta referring to the post-pre intervention moments) of the functional evaluation between the EG and CG.

\section{Effect Size}

Figure A and B present the effect size analysis for the cognitive and motor variables, respectively. The results have shown a better response in all the investigated variables in the exercise group, with greater effect size observed for the CAMCOG (1.17; 95\%CI, 0.21 to 2.12) and Stroop Test (1.14; 95\%CI, 0.19 to 2.09) (cognitive responses) and TUGT (1.58; $95 \% \mathrm{CI}, 0.56$ to 2.60$)$ and $\mathrm{FR}(1.48 ; 95 \% \mathrm{CI}, 0.48$ to 2.48) (motor responses).

\section{DISCUSSION}

The purpose of the present study was to evaluate the effect of a controlled moderate intensity aerobic exercise (treadmill training) on the cognitive and functional capacity of patients with $\mathrm{AD}$. There was a beneficial effect of the physical exercise on the global cognitive function CAMCOG, with a six-point improvement in EG and a six-point worsening in the CG. Also, there was a significant improvement in balance and mobility.

A number of recent studies have shown also show that physical exercise can improve cognitive performance. Kwak et al. ${ }^{10}$ observed a $30 \%$ improvement in overall cognitive state MMSE after 12 months of generalized exercise (balance training, strength, aerobic and flexibility). Another study ${ }^{11}$ demonstrated that the CG has declined three points in the MMSE as
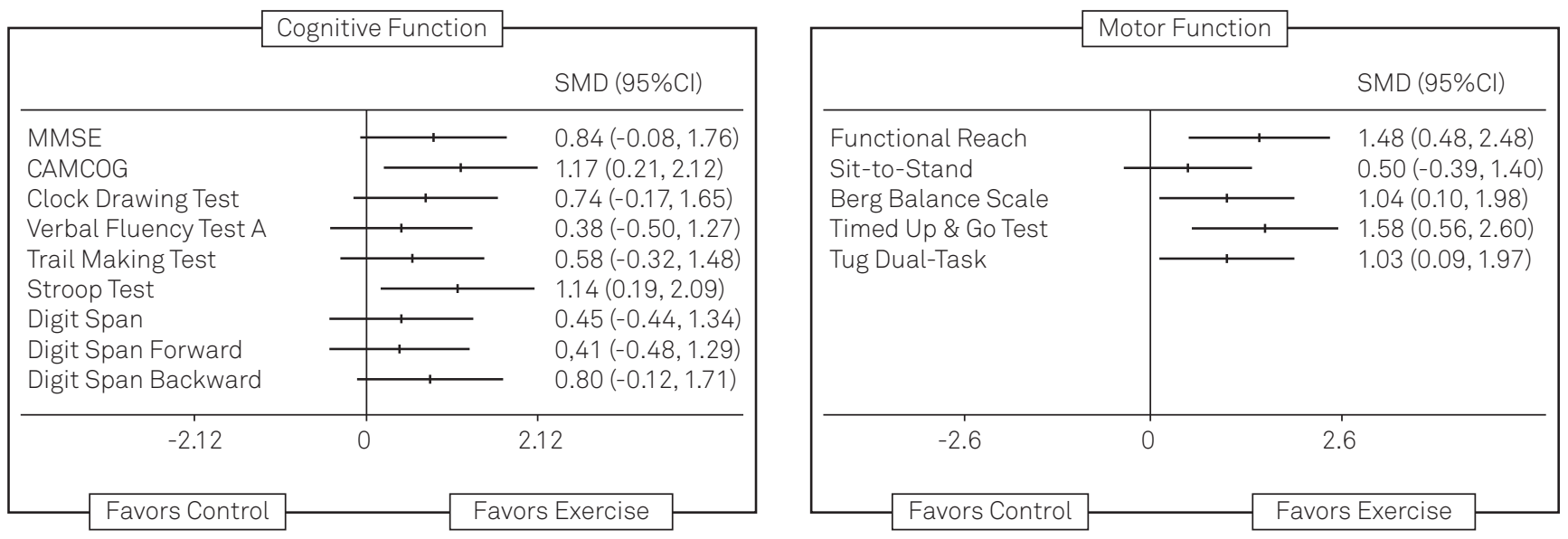

Figure. Effect size analysis after 4-month of intervention in both groups. Cognitive responses. MMSE: Mini-Mental State Examination; CAMCOG: Cambridge Cognitive Examination (A). Motor responses. SMD: standardized mean difference (B). 
compared to the EG. Several studies show that AD patients decrease a mean of three points on the MMSE per year ${ }^{26,27}$ and the two-point decrease might be an indication that there is a slower progression of the disease as a result of the pharmacological treatment ${ }^{27}$. Although we reached a marginal statistical significance on the MMSE results $(p=0.057)$, this may also be a trend to show that the practice of regular physical exercise might contribute to a slower decline. The CG lost two points whereas the EG maintained the baseline MMSE score. Noteworthy, both groups had been using anticholinesterase drugs for at least six months, and only the CG declined two points after the study. Our findings contrast with those reported by Eggermont et al. ${ }^{13}$ who did not observe significant differences in cognitive function of dementia patients who participated in walking tasks compared to a control group. A possible explanation for our positive results may reside in the fact that we used a controlled moderate intensity which allowed us to observe a dose-response effect of the aerobic exercise on the cognition of elderly with dementia. The beneficial effect of regular physical exercise on the cognitive function may be associated to a good performance of the functional capacity. There are consistent data from studies which show positive effects on the balance and fall risk after physical exercise in patients with dementia, including $\mathrm{AD}^{3,4,7,10,11}$. In our study, we have also found positive results on the balance and fall risk.

Walking time is considered a marker of the pre-clinical phase of the disease and for the risk of developing dementia as well ${ }^{28}$. Both EG and CG showed an excellent performance in the mobility test $(8 " \pm 2.6$ seconds; $8 " \pm 2.2$ seconds, respectively). Even so, it is noteworthy that the TUGT significantly improved in the EG compared to the CG. The evaluation of strength in the lower limbs did not present any statistically significant difference between the two groups $(\mathrm{p}=0.08)$. On the other hand, Santana-Sosa et al. ${ }^{7}$ have shown a significant improvement in the STS after 12 weeks of generalized exercise (balance, strength, flexibility and walking).
The most important neurophysiological hypotheses which attempt to explain cognitive improvement of elderly with $\mathrm{AD}$ are the increase and redistribution of cerebral blood flow, antioxidant action by repair enzymes and pro-inflammatory cytokines, beta-amyloid degradation, increased brain neurotrophic factors, neurogenesis, angiogenesis, and increase on the synthesis and metabolism of neurotransmitters ${ }^{29,30}$.

There are some limitations in this study which deserve mentioning. The small sample size $(n=20)$ is certainly a major issue and other studies should include larger samples. Likewise, the lack of a control group with the same social contact as the exercise group and the selection of patients with $\mathrm{AD}$ and $\mathrm{MD}$ may have influenced on the results.

This protocol has shown to be feasible and safe, with a high rate of adherence. Also it has provided favorable responses with regard to cognition and ADL, balance and other relevant measures. It is reasonable to propose that walking on the treadmill may be viewed as a more functional task than riding a bicycle, contributing to the improvement of functional transfers usually carried out in daily life. We propose that other such protocols should be used in future investigations in order to control intensity (moderate intensity of $60 \% \mathrm{VO}_{2 \max }$, duration (30 minutes), and frequency (twice a week), as suggested in the present study. It is important to emphasize that aerobic capacity represents only one among several domains of a training protocol. Exercise prescription must include other physical variables, such as strength, balance, flexibility, and coordination. The use of tools such as neuroimaging, invasive measurements (neurotransmitters, neurotrophic factors), or physiological markers associated to clinical diagnosis may further contribute to elucidate the effects of aerobic exercise in Alzheimer's disease.

\section{ACKNOWLEDGMENTS}

We thank Claudia Cresciula, MD, and Alexandre Rios, Professor, for their contributions to data collection.

\section{References}

1. Courtney C, Farrel D, Gray R, et al. Long-term donepezil treatment in 565 patients with Alzheimer's disease (AD2000): randomised doubleblind trial. Lancet 2004;363:2105-2115.

2. Hamer M, Chida Y. Physical activity and risk of neurodegenerative disease: a systemic review of prospective evidence. Psychol Med 2009;39:3-11.

3. Deslandes A, Moraes H, Ferreira C, et al. Exercise and mental health: many reasons to move. Neuropsychobiology 2009;59:191-198.

4. Arcoverde C, Deslandes A, Rangel A, et al. Role of physical activity on the maintenance of cognition and activities of daily living in elderly with Alzheimer's disease. Arq Neuropsiquiatr 2008;66:323-327.

5. Burns JM, Cronk BB, Anderson HS. Cardiorespiratory fitness and brain atrophy in early Alzheimer disease. Neurology 2008;71:210-216.
6. Rolland Y, Pillard F, Klapouszczak A. exercise program for nursing home residents with Alzheimer's disease: a 1- year randomised. Controlled trial. J Am Geriatr 2007;55:158-167.

7. Santana-Sosa E, Barriopedro MI, López-Mojares LM, Pérez M, Lucia A. Exercise training is beneficial for Alzheimer's patients. Int J Sports Med 2008;29:845-850.

8. Tappen RM, Williams CL, Barry C, Disesa D. Conversation intervention with Alzheimer's patients: increasing the relevance of communication. Clin Gerontol 2002;24:63-75.

9. Littbrand $H$, Stenvall M, Rosendahl E. Applicability and effects of physical exercise on physical and cognitive functions and activities of daily living among people with dementia: a systematic review. Am J Phys Med Rehabil 2011;90:495-518. 
10. Kwak YS, Um SY, Son TG, Kim DJ. Effect of regular exercise on senile dementia patients. Int J Sports Med 2008;29:471-474.

11. Hernandez SSS, Coelho FGM, Gobbi S, Stella F. Efeitos de um programa de atividade física nas funções cognitivas, equilíbrio e risco de quedas em idosos com demência de Alzheimer. Rev Bras Fisioter 2010;14:68-74.

12. Cott CA, Dawson P, Sidani S, Wells D. The effect of a walking/ talking program on commnucation, ambulation, and functional status in residents with Alzheimer disease. Alzheimer Dis Assoc Disord 2002:16:81-87.

13. Eggermont LHP, Swaab DF, Hol EM, Scherder EJA. Walking the line: a randomised Trial on the effects of a short term walking programme on cognition in dementia. J Neurol Neurosurg Psychiatry 2009;80:802-804

14. Forbes D, Forbes S, Morgan DG, Markle-Reid M, Wood J, Culum I. Physical activity programs for persons with dementia. Cochrane Database Syst Rev 2008;16:CD006489.

15. Radak Z, Chung HY, Goto S. Exercise and hormesis: oxidative stress-related adaptation for successful aging. Biogerontology 2005;6:71-75.

16. McKhann G, Drachman D, Folstein M, Katzman R, Price D, Stadlan EM. Clinical diagnosis of Alzheimer's disease: report of the NINCDSARDA work group under the auspices of the department of health and human services task force on Alzheimer's disease. Neurology 1984;34:939-944.

17. Román GC. Defining dementia: clinical criteria for the dignosis of vascular dementia. Acta Neurol Scand 2002;178(Suppl):S6-S9.

18. Folstein MF, Folstein SE, McHugh PR. Mini-mental state: a practical method for grading the cognitive state of patients for the clinician. J Psychiatric Res 1975;12:189-198.

19. Morris JC. The Clinical dementia rating (CDR): current version and scoring rules. Neurology 1993;43:2412-2414.
20. Alexopoulos GS, Abrams RC, Young RC, Shamoian CA. Cornell Scale for Depression in dementia. Biol Psychiatry 1998;23:271-284.

21. Bottino CMC, Almeida OP, Tamai S, Forlenza OV, Scalvo MZ, Carvalho IAM. Entrevista estruturada para o diagnóstico de transtornos mentais em idosos - CAMDEX. The Cambridge examination for mental disorders of the elderly. Brazilian version (translated and adapted on behalf of the editors). Cambridge: Cambridge University Press; 1999.

22. Wechsler D. Wechsler Adult Intelligence Scale - Revised manual (WAIS-R). New York: The Psychological Corporation; 1981.

23. Strauss E, Sherman EMS, Spreen O. A compendium of neuropshychological tests: administration, norms, and commentary. New York: Oxford University Press; 2006.

24. Berg KO, Norman KE. Functional assessment of balance and gait. Clin Geriatr Med 1996;12:705-723.

25. Rikli RE, Jones CJ. Senior fitness test manual. Champaign: Human Kinetics; 2001.

26. Salmon DP, Thai LJ, Butters N. Longitudinal evaluation of dementia of the Alzheimer type: a comparison of 3 standardized mental status examinations. Neurology 1990;40:1225-1230.

27. Lopez OL, Becker JT, Saxton J, Sweet R, Klunk W, Deosky ST. Alteration of a clinically meaningful outcome in the natural history of Alzheimer's disease by cholinesterase inhibition. J Am Geriatr Soc 2005;53:83-87.

28. Verghese J, Wang C, Lipton RB, Holtzer R, Xue X. Quantitative gait dysfunction and risk of cognitive decline and dementia. J Neurol Neurosurg Psychiatry 2007;78:929-935.

29. Radak Z, Hart N, Sarga L, et al. Exercise plays a preventive role against Alzheimer's disease. J Alzheimers Dis 2010;20:777-783.

30. Adlard PA, Perreau VM, Pop V, Cotman CW. Voluntary exercise decrease amyloid load in a transgenic model of Alzheimer's disease. J Neurosci 2005;25:4217-4221. 